Western University

Scholarship@Western

2015

\title{
Quantification of Cerebral Ventricle Volume Change of Preterm Neonates Using 3D Ultrasound Images
}

Yimin Chen

City University of Hong Kong

Jessica Kishimoto

Western University

Wu Qiu

Western University

Sandrine de Ribaupierre

Western University

Aaron Fenster

Western University

See next page for additional authors

Follow this and additional works at: https://ir.lib.uwo.ca/anatomypub

Part of the Anatomy Commons, and the Cell and Developmental Biology Commons

Citation of this paper:

Chen, Yimin; Kishimoto, Jessica; Qiu, Wu; de Ribaupierre, Sandrine; Fenster, Aaron; and Chiu, Bernard, "Quantification of Cerebral Ventricle Volume Change of Preterm Neonates Using 3D Ultrasound Images" (2015). Anatomy and Cell Biology Publications. 63.

https://ir.lib.uwo.ca/anatomypub/63 
Authors

Yimin Chen, Jessica Kishimoto, Wu Qiu, Sandrine de Ribaupierre, Aaron Fenster, and Bernard Chiu 


\section{Quantification of cerebral ventricle volume change of preterm neonates using 3D ultrasound images}

Yimin Chen

Jessica Kishimoto

Wu Qiu

Sandrine de Ribaupierre

Aaron Fenster

Bernard Chiu 


\title{
Quantification of Cerebral Ventricle Volume Change of Preterm Neonates Using 3D Ultrasound Images
}

\author{
Yimin Chen $^{a}$, Jessica Kishimoto ${ }^{b}$, Wu Qiu ${ }^{b}$, Sandrine de Ribaupierre ${ }^{c}$ and Aaron Fenster ${ }^{b}$, \\ Bernard Chiu ${ }^{a}$ \\ ${ }^{a}$ Department of Electronic Engineering, City University of Hong Kong, Hong Kong \\ ${ }^{b}$ Robarts Research Institute, Western University, London, Ontario, Canada \\ ${ }^{c}$ Department of Clinical Neurological Science, Western University, London, Ontario, Canada
}

\begin{abstract}
Intraventricular hemorrhage (IVH) is a major cause of brain injury in preterm neonates. Quantitative measurement of ventricular dilation or shrinkage is important for monitoring patients and in evaluation of treatment options. 3D ultrasound (US) has been used to monitor the ventricle volume as a biomarker for ventricular dilation. However, volumetric quantification does not provide information as to where dilation occurs. The location where dilation occurs may be related to specific neurological problems later in life. For example, posterior horn enlargement, with thinning of the corpus callosum and parietal white matter fibres, could be linked to poor visuo-spatial abilities seen in hydrocephalic children. In this work, we report on the development and application of a method used to analyze local surface change of the ventricles of preterm neonates with IVH from 3D US images. The technique is evaluated using manual segmentations from 3D US images acquired in two imaging sessions. The surfaces from baseline and follow-up were registered and then matched on a point-by-point basis. The distance between each pair of corresponding points served as an estimate of local surface change of the brain ventricle at each vertex. The measurements of local surface change were then superimposed on the ventricle surface to produce the 3D local surface change map that provide information on the spatio-temporal dilation pattern of brain ventricles following IVH. This tool can be used to monitor responses to different treatment options, and may provide important information for elucidating the deficiencies a patient will have later in life.
\end{abstract}

Keywords: Preterm neonate, cerebral ventricle, volume change, 3D ultrasound (US)

\section{INTRODUCTION}

Among very low birth weight, preterm neonates, the most common non-congenital cause of cerebral ventricle dilation is intraventricular hemorrhage (IVH). ${ }^{1}$ Post-hemorrhagic ventricle dilation (PHVD), especially PHVD requiring interventional therapies, such as ventricle tapping to remove excess cerebral spinal fluid (CSF) or a surgically inserted 'shunt' (as in the case of hydrocephalus), is linked to specific neuropsychological impairments, such as visuo-spatial and motor deficits. ${ }^{2}$ There are currently no means of accurately predicting the severity of PHVD or determining if and when ventricular dilation requires treatment. ${ }^{3}$ Decision on interventions is typically based on clinical signs such as increased head circumference, bulging of the anterior fontanelle, along with increases in ventricle size measured by ultrasound (US) as well as symptoms of increased intracranial pressure (ICP), the latter of which tend to be non-specific in this patient population. Monitoring PVHD in a quantitative manner may assist clinicians in theirs decisions on whether or not to intervene in patients with dilated ventricles.

$3 \mathrm{D}$ US has proven to be a reliable tool to measure the ventricle volume size ${ }^{4}{ }^{5}$ However, there are differential enlargement of specific parts of the ventricles over time, which cannot be identified by volume measurement alone. The analysis of local surface changes provides rich information that could explain some specific neurological and neuropsychological deficits seen in hydrocephalic patients such as those associated with visuo-spatial abilities and gross motor function ${ }^{6}{ }^{78}$ While hypotheses have been proposed about the importance of white matter integrity in the verbal and non-verbal developments of these hydrocephalic infants, there has been no studies, to our knowledge, on correlating the change of the ventricular shape segmented from 3D US to the deficits in intellectual developments of the hydrocephalic patients.

Medical Imaging 2015: Ultrasonic Imaging and Tomography, edited by Johan G. Bosch,

Neb Duric, Proc. of SPIE Vol. 9419, 94190V · (c) 2015 SPIE

CCC code: $1605-7422 / 15 / \$ 18 \cdot$ doi: $10.1117 / 12.2081470$ 


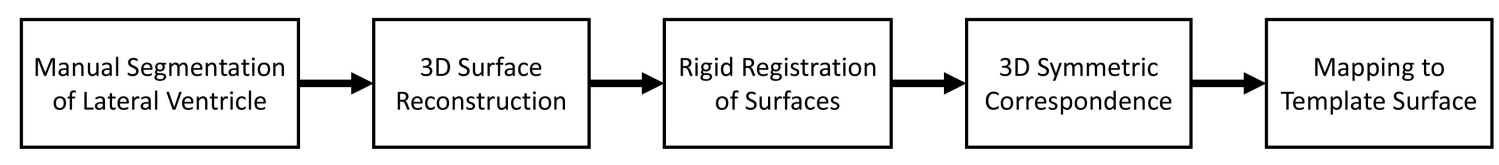

Figure 1. Schematic diagram of the proposed algorithm.

In this work, we developed a method for quantifying the spatio-temporal changes of the cerebral ventricle surfaces of IVH patients using 3D US. In addition, non-rigid point set registration was applied to map the proposed metric from different subjects together to make inter-subject comparison possible. Fig. 1 shows the schematic diagram of our proposed algorithm.

\section{METHODS}

\subsection{D US Image Acquisition}

3D US images were acquired with a motorized 3D US system developed for cranial scanning of preterm neonates in Neonatal Intensive Care Unit in University Hospital of The University of Western Ontario, which used an HDI 5000 (Philips, Bothel WA) and C8-5 (Philips, Bothel WA) curved array 5-8 MHz broadband transducer. ${ }^{5}$ To perform a scan, an US technician located the third ventricle, midline through the anterior fontanelle with the patient inside an incubator, and then the 3D US system mechanically tilted the $2 \mathrm{D}$ transducer to acquire a full $3 \mathrm{D}$ image of the ventricular system. The $3 \mathrm{D}$ image sizes ranged from $300 \times 300 \times 300$ to $450 \times 450 \times 450$ voxels with a voxel size of $0.22 \times 0.22 \times 0.22 \mathrm{~mm}^{3}$.

\subsection{Manual Segmentation and 3D Surface Reconstruction}

The cerebral lateral ventricles were manually segmented on a slice-by-slice basis using an in-house $3 \mathrm{D}$ viewing software (3D Quantify, Robarts Research Institute, The University of Western Ontario, London, Canada). A trained observer blinded to the image orders segmented each subject in parallel slices with $1 \mathrm{~mm}$ interval. These planar contours are reconstructed into a 3D surface based on the distance map of each contour. ${ }^{9}$ Specifically, a map showing the shortest signed distance from each point to the contour was generated in each transverse image. Points inside and outside the contour were respectively equipped with positive and negative values. Distance values at points between two adjacent transverse images were obtained by linearly interpolating distance maps on the transverse images. A marching cube algorithm was applied to extract zero level set of the distance map to generate the $3 \mathrm{D}$ surface. ${ }^{10}$

\subsection{D Surface Change $(\Delta S)$ Map}

To compute the local difference between surfaces obtained at baseline and follow-up from the same subject, the iterative closest point (ICP) algorithm was applied to align two surfaces as shown in Fig. 2. Then, a 3D symmetric correspondence algorithm ${ }^{11}$ was used to match these two registered surfaces on a point-by-point basis. Specifically, the symmetric nearest neighbor correspondences between those two surfaces were established first and then an interpolation process was applied to find the corresponding points for the points without symmetric nearest points. After that, a smoothing procedure was used to smooth those displacement fields connecting pairs of correspondence points. For each pair of corresponding points connected by the green arrow as shown in blue rectangle box of Fig. 2, we computed signed distance $(\Delta S)$ between them, with positive values for surface dilation and negative values for surface shrinkage.

\subsection{Mapping to the template shape}

Although $\Delta S$ can be mapped to the ventricle surface of a subject (3D $\Delta S$ map) to facilitate visualization and interpretation, the shape of the 3D $\Delta S$ map is highly subject-specific. Quantitative point-by-point comparison of $\Delta S$ between subjects was therefore not possible. To address this issue, a robust point set registration method known as coherent point drift (CPD) algorithm ${ }^{12}$ was applied to match the $3 \mathrm{D} \Delta S$ maps of different subjects 


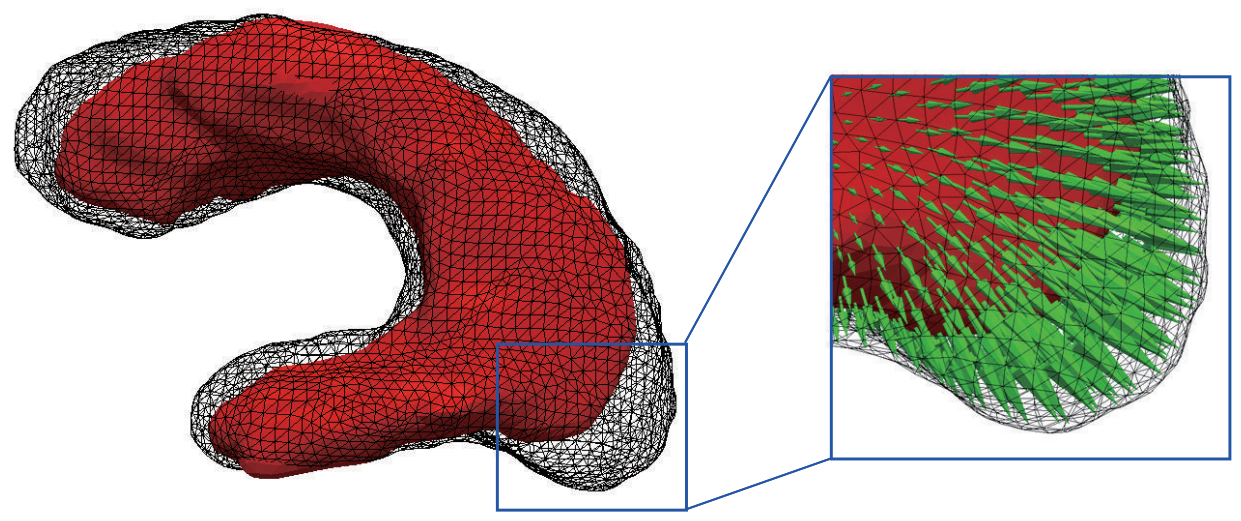

Figure 2. Rigid registration of the left cerebral ventricle surfaces of a subject with grade III IVH obtained at baseline and follow-up (red solid: surface at baseline and black mesh: surface at follow-up). Green arrows connect pairs of corresponding points.

to a template surface (selected from a set of surfaces from 3D US by a physician). Considering the point set $X=\left(x_{1}, \ldots, x_{N}\right) \in \mathbb{R}^{N \times D}$ of template surface and point set $Y=\left(y_{1}, \ldots, y_{M}\right) \in \mathbb{R}^{M \times D}$ of subject surface, we aimed to find a transformation $f$ to make that:

$$
f: X=Y+f(Y)
$$

And a Gaussian mixture model (GMM) listed as below was established as the likelihood function to obtain the optimal transformation:

$$
p(X \mid Y, \theta)=\prod_{n=1}^{N} p\left(x_{n} \mid Y, \theta\right)=\prod_{n=1}^{N} \sum_{m=1}^{M} \frac{\pi_{m}}{\left(2 \pi \sigma^{2}\right)^{D / 2}} e^{-\frac{\left\|x_{n}-y_{m}-f\left(y_{m}\right)\right\|^{2}}{2 \sigma^{2}}}
$$

where $\theta=\left\{f, \pi_{m}, \sigma^{2}\right\}$ is a set of unknown model parameters. $\pi_{m}$ is the prior probability and $\sigma^{2}$ represents the isotropic variance of all GMM components. Expectation-Maximization was applied to maximize the likelihood function and move the GMM centroids (subject surface) to point set of template surface as group coherently to preserve the topological structure of the point set.

Based on the registered surfaces from the CPD, we applied the 3D symmetric correspondence algorithm described in Sec. 2.3 to generate an accurate point-by-point correspondence between the template and subject surface. As a result, the 3D $\Delta S$ map of each subject was mapped onto the template surface, making point-bypoint comparison between $3 \mathrm{D} \Delta S$ maps of different subjects possible.

\section{RESULTS}

We used cerebral ventricle 3D US images acquired from two subjects at two scanning sessions to verify the performance of our algorithm and to demonstrate its application. Subject 1 diagnosed with severe IVH (grade III bilateral ${ }^{13}$ ) was not treated between two scanning sessions that were 3 days apart and ventricle dilation was expected. Table 1 shows the volume measurements of left and right lateral ventricle (LLV and RLV) at baseline and follow-up. Both the left and right ventricle of this subject show an increase in volume after three days. In particular, the volume of left ventricle increased by approximately 55\%. Fig. 3 (a) and (b) show the histograms of point-wise $\Delta S$ for LLV and RLV of Subject 1 respectively. Fig. 4 (a) shows the ventricle surface of this subject at baseline with local surface change color-coded and superimposed. Point-wise $\Delta S$ was mapped to the template surface and displayed in Fig. 4 (b). We observed that the largest dilation was located in the posterior horn region of the ventricle. For Subject 2, the left lateral ventricle had been drained of CSF during a tapping procedure after the patient had become clinically symptomatic for raised ICP. 3D ultrasound images before and after the 


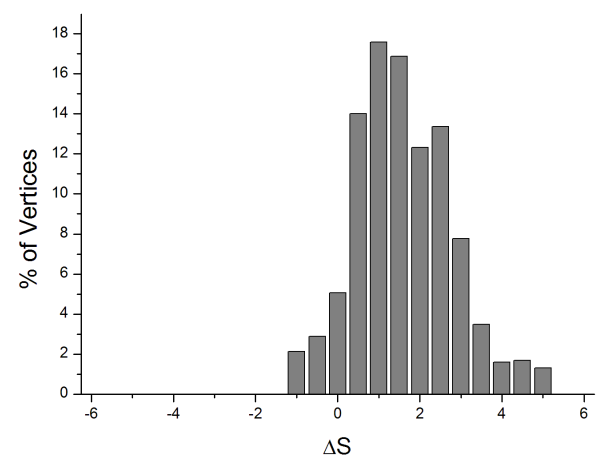

(a)

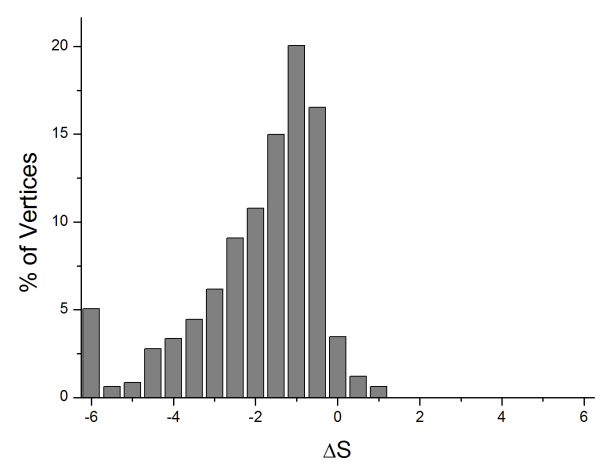

(c)

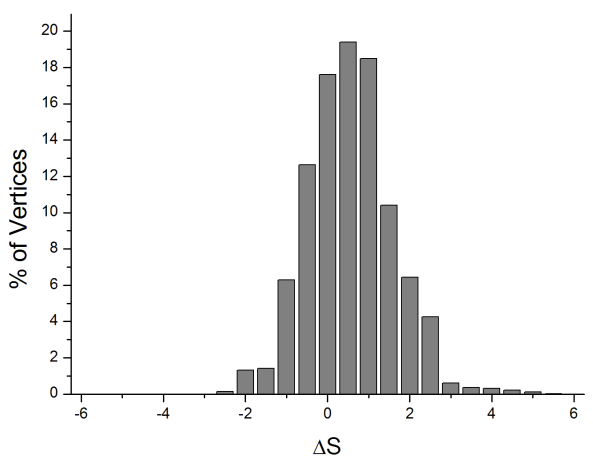

(b)

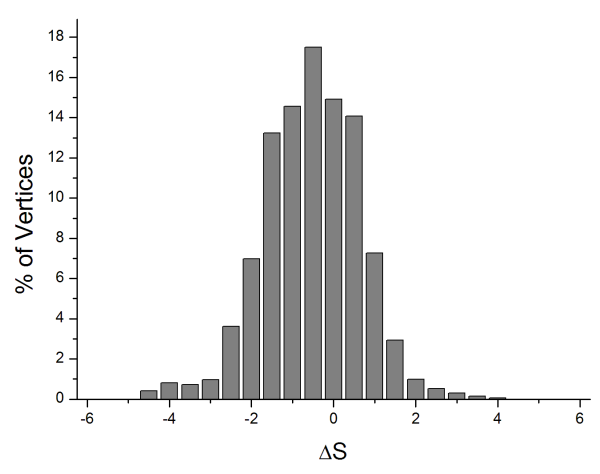

(d)

Figure 3. Frequency distribution of $\Delta S$ for two subjects. (a) and (b) are the $\Delta S$ distribution for LLV and RLV of Subject 1 respectively. (c) and (d) are the $\Delta S$ distribution for LLV and RLV of Subject 2 respectively.

tapping operation were acquired. As all ventricles are connected through the 3rd ventricle, by tapping the left lateral ventricle it was hypothesized to reduce the volume of both lateral ventricles. The volume measurements listed in Table 1 and distribution of $\Delta S$ for LLV and RLV shown in Fig. 3 (c) and (d) support this hypothesis. However, it was also observed that the amount of shrinkage is much larger in the left ventricle than in the right ventricle as shown in Fig. 5. In particular, Fig. 5 shows the difference in the amount of shrinkage for the left and right ventricles is the most prominent in the neighbourhood of the posterior horn. This is likely due to the fact that the repeat US was acquired a few minutes only after the tap, and that the hemorrhage in the ventricles caused an increase in the viscosity of the CSF as well as obstacles in the circulation of CSF with in the ventricles.

\begin{tabular}{|c|c|c|c|c|}
\hline & \multicolumn{2}{|c|}{ Baseline } & \multicolumn{2}{|c|}{ Follow-up } \\
\hline & $\overline{\mathrm{LLV}}$ Volume $\left(\mathrm{cm}^{3}\right)$ & RLV Volume $\left(\mathrm{cm}^{3}\right)$ & $\overline{\text { LLV Volume }\left(\mathrm{cm}^{3}\right)}$ & RLV Volume $\left(\mathrm{cm}^{3}\right)$ \\
\hline Subject 1 & 6.84 & 7.03 & 10.61 & 8.90 \\
\hline Subject 2 & 18.44 & 24.61 & 11.42 & 22.41 \\
\hline
\end{tabular}

Table 1. Volume measurement of patients lateral ventricles at baseline and follow-up. LLV is the left lateral ventricle and RLV is the right lateral ventricle.

\section{DISCUSSION}

We developed a technology to monitor the spatio-temporal surface change of the lateral ventricles using 3D US. This tool has the ability to identify regions where large changes occur in subjects with untreated IVH and after surgical intervention. To allow inter-subject comparison in local ventricular change, we applied non-rigid point 


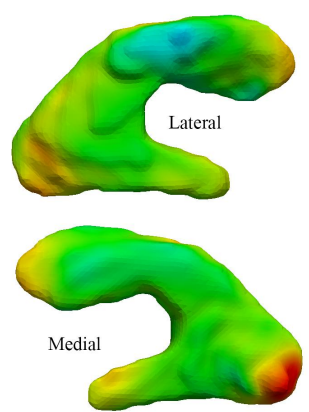

Right
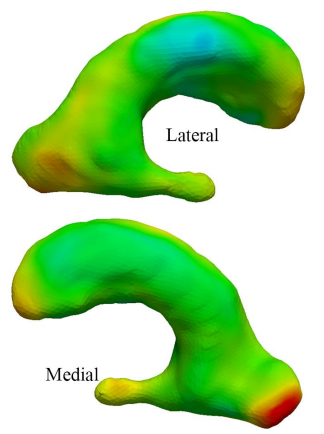

Right

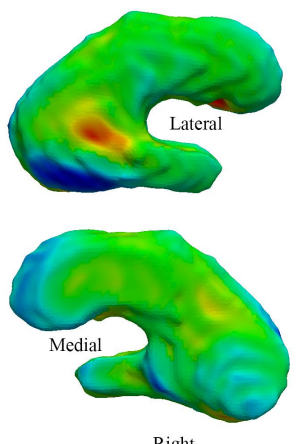

Right
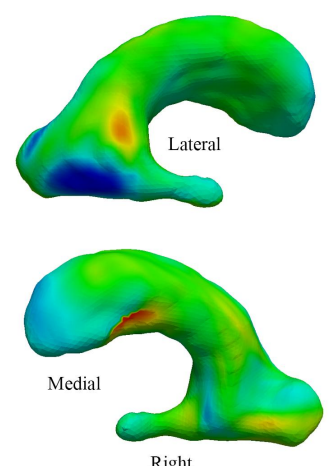

Right

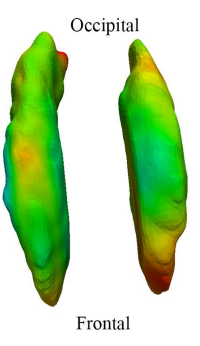

(a)

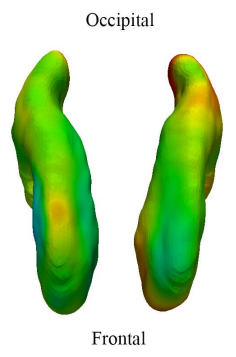

(b)

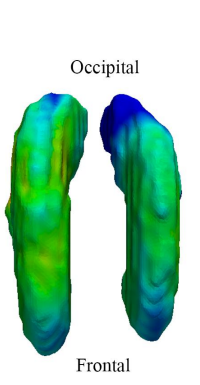

(a)

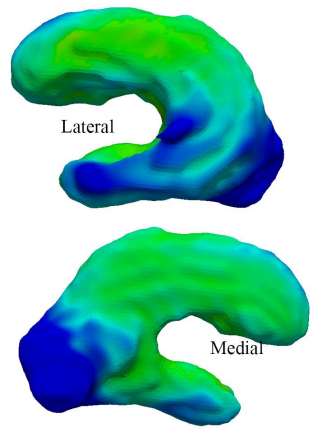

Left
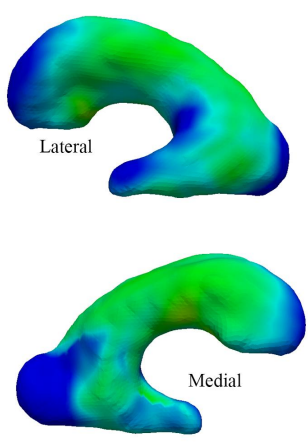

Left
$\Delta \mathrm{S}(\mathrm{mm})$
$\mathrm{n}$

Figure 4. (a) Subject 1 and (b) template surface with local surface change color-coded and superimposed. The patient had bilateral grade III IVH, and was expected to undergo ventricle dilation over the first weeks of life. Images were acquired three days apart and surfaces maps show the expected dilation mostly in the posterior horn, as well as the frontal horns (red).

Figure 5. (a) Subject 2 who had a tapping procedure and (b) template surface with local surface change color-coded and super-imposed. The patient had diagnosed bilateral grade III IVH and required a ventricle tap. The left lateral ventricle was tapped, and showed an expected decrease in ventricle size (blue) as shown on the surface maps; however, the right ventricle didn't decrease as much, due to partial obstruction of the pathways caused by an intraventricular hemorrhage. 
set registration (CPD) and 3D symmetric correspondence to map surfaces of different subjects to a template surface on point-by-point basis. This approach has the potential to help correlate localized damage (caused by excessive ventricle enlargement) to neurological problems later in life.

There were several limitations in this study. First, manual segmentation of the 3D ventricular images of neonates is labor intensive and has been shown to associate with high segmentation variability. ${ }^{14}$ Although a semi-automatic algorithm has been proposed, ${ }^{14}$ until the local segmentation accuracy of this algorithm has been thoroughly validated, manual segmentation is still regarded as the most reliable technique in delineating local structures of the ventricle. Second, the quantification of local change is based on a single segmentation on each of the baseline and follow-up images. As manual segmentation is prone to variability, repeated segmentations may be required to establish statistically whether the computed surface change is due to real biological change or appears merely because of the segmentation variability.

\section{CONCLUSIONS}

In this paper, we described a technology to monitor the spatio-temporal surface change of cerebral ventricles and its application to model the surface change of preterm neonate's ventricles with untreated IVH and after surgical intervention. The metric of local surface change was defined as the signed distance between the pair of corresponding points. We demonstrated the use of our algorithm to quantify local surface change for two subjects involved in this study.

\section{ACKNOWLEDGMENTS}

This study is supported by a grant from the Research Grant Council of the HKSAR, China (Project No. CityU 139713) and the National Natural Science Foundation of China (Grant No. 81201149).

\section{REFERENCES}

[1] A. R. Synnes, L.-Y. Chien, A. Peliowski, R. Baboolal, and S. K. Lee, "Variations in intraventricular hemorrhage incidence rates among canadian neonatal intensive care units," Journal of Pediatrics 138(4), pp. 525$531,2001$.

[2] M. Mataró, C. Junqué, M. A. Poca, and J. Sahuquillo, "Neuropsychological findings in congenital and acquired childhood hydrocephalus," Neuropsychology Review 11(4), pp. 169-178, 2001.

[3] L. S. de Vries, A. J. Brouwer, and F. Groenendaal, "Posthaemorrhagic ventricular dilatation: when should we intervene?," Archives of Disease in Childhood-Fetal and Neonatal Edition 98(4), pp. F284-F285, 2013.

[4] J. H. Gilmore, G. Gerig, B. Specter, H. C. Charles, J. S. Wilber, B. S. Hertzberg, and M. A. Kliewer, "Infant cerebral ventricle volume: a comparison of 3-D ultrasound and magnetic resonance imaging," Ultrasound Med. Biol. 27(8), pp. 1143-1146, 2001.

[5] J. Kishimoto, S. de Ribaupierre, D. Lee, R. Mehta, K. St Lawrence, and A. Fenster, "3D ultrasound system to investigate intraventricular hemorrhage in preterm neonates," Phys. Med. Biol. 58(21), p. 7513, 2013.

[6] J. Donders, B. P. Rourke, and A. I. Canady, "Neuropsychological functioning of hydrocephalic children," Journal of Clinical and Experimental Neuropsychology 13(4), pp. 607-613, 1991.

[7] J. M. Fletcher, D. J. Francis, N. M. Thompson, B. L. Brookshire, T. P. Bohan, S. H. Landry, K. C. Davidson, and M. E. Miner, "Verbal and nonverbal skill discrepancies in hydrocephalic children," Journal of Clinical and Experimental Neuropsychology 14(4), pp. 593-609, 1992.

[8] J. Hodel, P. Besson, A. Rahmouni, E. Petit, A. Lebret, B. Grandjacques, O. Outteryck, M. A. Benadjaoud, A. Maraval, A. Luciani, et al., "3D mapping of cerebrospinal fluid local volume changes in patients with hydrocephalus treated by surgery: preliminary study," European radiology 24(1), pp. 136-142, 2014.

[9] A. G. Bors, L. Kechagias, and I. Pitas, "Binary morphological shape-based interpolation applied to 3-D tooth reconstruction," Medical Imaging, IEEE Transactions on 21(2), pp. 100-108, 2002.

[10] W. E. Lorensen and H. E. Cline, "Marching cubes: A high resolution 3D surface construction algorithm," in ACM Siggraph Computer Graphics, 21(4), pp. 163-169, ACM, 1987. 
[11] X. Papademetris, A. J. Sinusas, D. P. Dione, R. T. Constable, and J. S. Duncan, "Estimation of 3-D left ventricular deformation from medical images using biomechanical models," Medical Imaging, IEEE Transactions on $\mathbf{2 1}(7)$, pp. 786-800, 2002.

[12] A. Myronenko and X. Song, "Point set registration: Coherent point drift," Pattern Analysis and Machine Intelligence, IEEE Transactions on 32(12), pp. 2262-2275, 2010.

[13] L.-A. Papile, J. Burstein, R. Burstein, and H. Koffler, "Incidence and evolution of subependymal and intraventricular hemorrhage: a study of infants with birth weights less than 1,500 gm," The Journal of Pediatrics 92(4), pp. 529-534, 1978.

[14] W. Qiu, J. Yuan, J. Kishimoto, J. McLeod, Y. Chen, S. de Ribaupierre, and A. Fenster, "User-guided segmentation of preterm neonate ventricular system from 3-D ultrasound images using convex optimization," Ultrasound Med. Biol. 20, pp. 542-556, 2014. 